\title{
Tanshinol alleviates impaired bone formation by inhibiting adipogenesis via KLF15/PPARY2 signaling in GIO rats
}

\author{
Ya-jun YANG ${ }^{1}$, Zhu ZHU ${ }^{2}$, Dong-tao WANG ${ }^{3}$, Xin-le ZHANG ${ }^{1}$, Yu-yu LIU ${ }^{1}$, Wen-xiu LAI ${ }^{1}$, Yu-lin MO ${ }^{1}$, Jin LI $^{1}$, Yan-long LIANG $^{1}$, \\ Zhuo-qing $\mathrm{HU}^{1}$, Yong-jie $\mathrm{YU}^{1}$, Liao $\mathrm{CU}^{1,4, *}$ \\ ${ }^{1}$ Department of Pharmacology, Guangdong Medical University, Zhanjiang 524023, China; ${ }^{2}$ Sino-American Cancer Research Institute, \\ Guangdong Medical University, Dongguan 523808, China; ${ }^{3}$ Department of Traditional Chinese Medicine, Shenzhen Hospital, Southern \\ Medical University, Shenzhen 518000, China; ${ }^{4}$ Guangdong Key Laboratory for R\&D of Natural Drugs, Guangdong Medical University, \\ Zhanjiang 524023, China
}

\begin{abstract}
Glucocorticoid (GC)-induced osteoporosis (GIO) is characterized by impaired bone formation, which can be alleviated by tanshinol, an aqueous polyphenol isolated from Salvia miltiorrhiza Bunge. In this study we investigated the molecular mechanisms underlying GC-induced modulation of osteogenesis as well as the possibility of using tanshinol to interfere with GIO. Female SD rats aged 4 months were orally administered distilled water (Con), prednisone (GC, $\left.5 \mathrm{mg} \cdot \mathrm{kg}^{-1} \cdot \mathrm{d}^{-1}\right)$, GC plus tanshinol (Tan, $\left.16 \mathrm{mg} \cdot \mathrm{kg}^{-1} \cdot \mathrm{d}^{-1}\right)$ or GC plus resveratrol (Res, $5 \mathrm{mg} \cdot \mathrm{kg}^{-1} \cdot \mathrm{d}^{-1}$ ) for 14 weeks. After the rats were sacrificed, samples of bone tissues were collected. The changes in bone formation were assessed using Micro-CT, histomorphometry, and biomechanical assays. Expression of Kruppel-like factor 15 (KLF15), peroxisome proliferator-activated receptor y 2 (PPARy 2) and other signaling proteins in skeletal tissue was measured with Western blotting and quantitative RT-PCR. GC treatment markedly increased the expression of KLF15, PPARY2, C/EBP $\alpha$ and aP2, which were related to adipogenesis, upregulated FoxO3a pathway proteins (FoxO3a and Gadd45a), and suppressed the canonical Wnt signaling ( $\beta$-catenin and Axin2), which was required for osteogenesis. Thus, GC significantly decreased bone mass and bone quality. Co-treatment with Tan or Res effectively counteracted GC-impaired bone formation, suppressed GC-induced adipogenesis, and restored abnormal expression of the signaling molecules in GIO rats. We conclude that tanshinol counteracts GC-decreased bone formation by inhibiting marrow adiposity via the KLF15/PPARY2/Fox03a/Wnt pathway.
\end{abstract}

Keywords: glucocorticoid-induced osteoporosis (GIO); tanshinol; resveratrol; bone formation; Kruppel-like factor 15 (KLF 15); PPARY 2; adipogenesis

Acta Pharmacologica Sinica (2018) 39: 633-641; doi: 10.1038/aps.2017.134; published online 11 Jan 2018

\section{Introduction}

Glucocorticoid-induced osteoporosis (GIO) is a common complication of chronic noninfectious inflammatory diseases during long-term glucocorticoid (GC) therapy ${ }^{[1]}$. A characteristic feature of GIO is marked impairment of new bone formation with nonspecific clinical symptoms ${ }^{[2]}$. Thus, there is a pressing need to search for novel treatment strategies for GIO based on new findings linked to bone formation.

Generally, bone formation requires a large number of mature osteoblastic cells during bone remodeling. It was reported that functional decline of bone marrow mesenchymal

\footnotetext{
* To whom correspondence should be addressed.

E-mail cuiliao@163.com (Liao CUI)

Received 2017-06-12 Accepted 2017-09-18
}

stem cells (BMSCs) induces impaired osteogenic potential with a misdirected differentiation toward adipocytes, contributing to osteoporotic bone loss accompanied by increased marrow adiposity ${ }^{[3]}$. A substantial number of therapeutic drugs directly promote adipogenesis at the expense of osteogenesis, especially corticosteroids ${ }^{[4]}$. Previous evidence has revealed that BMSCs treated with dexamethasone (Dex) are not committed to osteogenic differentiation but to adipogenic differentiation $^{[5]}$. Our previous study confirmed that prednisone acetate contributes to the increased adipocyte distribution in cancellous bone in the femur of rats ${ }^{[6]}$. However, how GC influence the balance between osteogenesis and adipogenesis in the pathological process of osteoporosis has not been studied thoroughly.

Recent findings have demonstrated that adipogenesis can 
be regulated in MSCs by peroxisome proliferator activated receptor $\gamma$ (PPARY, a master regulator of adipogenesis) at the expense of the osteogenesis required for Wnt signaling pathway ${ }^{[3,7,8]}$. Other evidence has further verified that activation of PPARY alters the fate of MSCs via suppression of osteoblastic differentiation ${ }^{[8,9]}$. Furthermore, the coordination of PPARY with the CCAAT/enhancer-binding protein a $(\mathrm{C} / \mathrm{EBPa})$ transcription factors controls the terminal differentiation of adipocytes and maintains adipocyte gene expression ${ }^{[7,8]}$. Meanwhile, activation of PPARY induces C/EBPa expression, and vice versa.

A novel identified glucocorticoid receptor (GR) target gene named Kruppel-like factor 15 (KLF) 15 is involved in adipogenesis $^{[10]}$. In MSCs, Dex induces activation of the KLF15 transcription factor, followed by increased expression of PPAR $\gamma$, $\mathrm{C} / \mathrm{EBPa}$ and adipocyte fatty acid binding protein 2 (aP2, an adipocyte-specific gene), which contributes to adipocyte differentiation ${ }^{[11,12]}$. Conversely, adipogenesis can be suppressed in part through inhibition of PPAR 2, C/EBPa and/or KLF15 expression ${ }^{[13-15]}$. Additionally, our previous evidence confirmed that GC-elicited activation of KLF15 repressed bone formation and subsequent bone loss in vivo via down-regulation of the Wnt signaling pathway ${ }^{[16]}$. Moreover, KLF15 triggered by GC can stimulate expression of the forkhead box $\mathrm{O}$ 3a (FoxO3a) transcription factor in skeletal muscle ${ }^{[17]}$. Another of our previous in vitro studies demonstrated that inhibition of FoxO3a promotes osteoblast differentiation via up-regulation of the Wnt pathway ${ }^{[18]}$. Whether activation of KLF15 in response to a high-dose of GC leads to an imbalance between osteogenesis and adipogenesis and subsequently contributes to osteoporosis remains unclear.

Tanshinol $(D(+) \beta-3,4$-dihydroxyphenyl lactic acid, also called danshensu) is an aqueous polyphenol isolated from Salvia miltiorrhiza Bunge (Figure 1$)^{[19]}$. Our previous evidence demonstrated that tanshinol exerts a protective effect on bone formation either in zebrafish and rats or in MSCs exposed to excessive GC ${ }^{[6,20,21]}$. Moreover, our previous findings confirmed that tanshinol regulates the Wnt/FoxO3a signaling pathway, contributing to the suppression of decreased osteogenesis in vitro under oxidative stress, similar to resveratrol (positive control), which has a chemical structure similar to that of tanshino ${ }^{[18]}$. Our data further revealed that tanshinol prevented bone loss by inhibiting the activation of KLF15 elicited by GC and subsequently blocking down-regulation of the Wnt pathway in GIO model rats ${ }^{[16]}$. However, the exact molecular mechanism of tanshinol underlying its ability to inhibit impaired bone formation induced by GC has not been

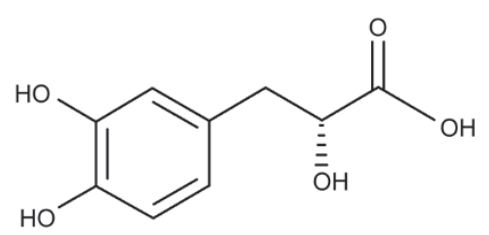

Figure 1. Structure of tanshinol. thoroughly investigated. Particularly, how tanshinol suppresses activation of the KLF15 cascade pathway and whether the osteo-protective effect of tanshinol is involved in rescuing the imbalance between adipogenesis and osteogenesis remain unclear.

Based on the evidence mentioned above, we hypothesized that tanshinol could prevent GC-elicited bone loss through inhibition of adipogenesis via the KLF15/PPARY2/FoxO3a/ Wnt cascade pathway. Hence, in the present study, we attempted to investigate the inhibitory effects of tanshinol on adipogenesis and its stimulatory actions on osteogenesis in a rat model of GIO. In addition, we focused on the underlying molecular mechanism that tanshinol may employ to regulate the KLF15 pathway and downstream signaling molecules, including PPARy2, C/EBPa, aP2, and FoxO3a, and to potentially impact Wnt signaling, which is required for osteogenesis.

\section{Materials and methods \\ Animal experiments}

Four-month-old female Sprague-Dawley rats $(225 \pm 25 \mathrm{~g}, n=32)$ were purchased from the Center of Experimental Animals of Sun Yat-sen University Ltd (certificate of quality: SCXK (YUE) 2012-0112). The animals were housed in Guangdong Medical University according to the recommendations in the Guide for the Care and Use of Laboratory Animals of Guangdong Laboratory Animal Monitoring Institute. All experimental methods were approved by the Academic Committee on the Ethics of Animal Experiments of the Guangdong Medical University (Permit Number: SYXK (YUE) 2008-0007). All rats were fed standard chow, given free access to water, and maintained at an appropriate temperature $\left(25 \pm 1^{\circ} \mathrm{C}\right)$ with a humidity level of $70 \%$ and a 12-h light-dark cycle. Rats were randomly assigned to the following four groups: Con, standard chow and distilled water $(n=8) ; \mathrm{GC}$, prednisone acetate $5 \mathrm{mg} \cdot \mathrm{kg}^{-1} \cdot \mathrm{d}^{-1}(n=8)$; Tan+GC, GC plus Tanshinol $16 \mathrm{mg} \cdot \mathrm{kg}^{-1} \cdot \mathrm{d}^{-1}(n=8)$; and Res+GC, GC plus Resveratrol $5 \mathrm{mg} \cdot \mathrm{kg}^{-1} \cdot \mathrm{d}^{-1}(n=8)$. All rats except those in the Con group were orally administered prednisone acetate in the morning and given other drugs in the afternoon once a day over a period of 14 weeks. All animals were injected subcutaneously with calcein $(10 \mathrm{mg} / \mathrm{kg}$, Sigma, St Louis, MO, USA) on days 13 and 14 as well as on days 3 and 4 before sacrifice. All rats were weighed once per week.

\section{Sample collection and applications}

All rats were sacrificed at the experimental end point. The right femurs were collected for evaluation of the bone biomechanical features and bone micro-architecture. The proximal metaphysis of the right tibia was prepared as an undecalcified section for bone histomorphometry. The left tibias were prepared to detect gene and protein expression.

\section{Bone histological and structural analysis}

For histomorphometric detection, the tibia was fixed in $10 \%$ phosphate-buffered formalin for $24 \mathrm{~h}$, followed by dehydration in an ascending ethanol series, and then embedded 
undecalcified in methyl methacrylate. The tissues were cut into 5-mm sections and stained with von Kossa to observe the trabecular architectural properties, including the trabecular bone area (\% Tb.Ar), trabecular number ( $\mathrm{Tb} . \mathrm{N})$, and increase in trabecular spacing ( $\mathrm{Tb} . \mathrm{Sp}$ ), and 9-mm sections were left unstained to examine the indicated fluorescence labels to evaluate the indices of bone formation, such as the bone formation rate/bone surface (BFR/BS), bone formation rate/tissue volume (BFR/TV) and percent of osteoblast perimeter (\%Ob.Pm), by virtue of two fluorescent labels. Histomorphometric assays were performed using Osteomeasure software (OsteoMetrics, Decatur, GA, USA). The bone trabecular micro-architecture of cancellous bone in the right proximal femur was determined using micro-computed tomography (Micro-CT, SCANCO vivaCT40, Basserdorf, Switzerland). In brief, femurs were placed vertically in 12-mm-diameter scanning holders and scanned at a $50-\mathrm{kVp}$ energy and $160-\mu \mathrm{A}$ intensity, with an integration time of $200 \mathrm{~ms}$ and voxel size of $18 \mu \mathrm{m}$. Trabecular $3 \mathrm{D}$ images of the region of interest (ROI) were reconstructed with a threshold of 190 at 1-4 mm distal to the growth plateepiphyseal junctions.

\section{Three-point bending test}

The mechanical strength of the long bone was measured with a three-point bending test using testing machinery (MTS, Eden prairie, Minnesota, USA). The frozen right femurs were thawed at room temperature and tested with a 1-mm indenter at a speed of $2 \mathrm{~mm} / \mathrm{min}$ with a $15-\mathrm{mm}$ span (L). Elastic load $(\mathrm{N})$, fracture load $(\mathrm{N})$, bending energy $(\mathrm{N} \times \mathrm{mm})$ and stiffness $\left(\mathrm{N} \times \mathrm{mm}^{2}\right)$ were obtained by calculation according to a loaddeformation curve.

\section{Quantitative RT-PCR detection}

The frozen left tibias were crushed in liquid nitrogen, and total RNA was extracted from bone powder using peqGOLD TriFast (Peqlab, Erlangen, Germany) according to the manufacturer's protocol. To obtain cDNA, equal amounts of total RNA were reverse-transcribed into cDNA using Superscript II Reverse Transcriptase (Invitrogen, Darmstadt, Germany). Real-time RT-PCR was performed in a 20 -mL reaction mixture containing $10 \mathrm{~mL}$ of SYBR Green Master Mix (Applied Biosystems, Carlsbad, CA, USA), 10 pmol of forward primer, 10 pmol of reverse primer, and $1 \mu \mathrm{g}$ of cDNA using a Stratagene Mx3005P QPCR System (La Jolla, CA, USA). The primer sequences are listed in Table 1 . The PCR results were analyzed using Opticon Monitor Analysis 2.0 software (Bio-Rad Laboratories, Hercules, CA, USA). Relative mRNA expression was calculated by subtracting the glyceraldehyde 3-phosphate dehydrogenase (GAPDH) threshold cycle $\left(C_{t}\right)$ value from the $C_{t}$ value of the genes of interest, and the data are expressed as $2^{-\Delta \Delta C t}$, as described by the protocol of the manufacturer.

\section{Western blotting assay}

For Western blotting, bone tissues were lysed in ice-cold RIPA buffer containing a complete protease inhibitor cocktail. The protein concentration was determined using a BCA protein
Table 1. Primers used for semiquantitative analyses of gene expression.

\begin{tabular}{llll}
\hline Genes & Species & \multicolumn{2}{c}{ Primer sequences } \\
& & Forward primer $\left(5^{\prime}-3^{\prime}\right)$ & Reverse primer $\left(5^{\prime}-3^{\prime}\right)$ \\
\hline C/EBP $\alpha$ & Rat & AGCAACGAGTACCGGGTACG & TGTTTGGCTTTATCTCGGCTC \\
aP2 & Rat & AGAAGTGGGAGTTGCTTCG & ACTCTCTGACCGGATGACGA \\
Gadd45 & Rat & ATCGAAAGGATGGACACGGT & TGAATGTGGTTCGTCACCA \\
PPARY2 & Rat & CTCCTGTTGACCCAGAGCAT & CAACCATTGGGTCAGCTCTT \\
KLF15 & Rat & TCCTCCAACTTGAACCTGTC & CTTGGTGTACATCTTGCTGC \\
Axin2 & Rat & AGTCAGCAGAGGGACAGGA & CTTGGAGTGCGTGGACACTA \\
GAPDH & Rat & CCATCATGAAGTGTGACGTG & ACATCTGCTGGAAGGGGAC
\end{tabular}

assay kit. Western blotting was performed as described in detail ${ }^{[16]}$. Antibodies recognizing KLF15, $\beta$-catenin, and FoxO3a were purchased from Cell Signaling Technology (Beverly, MA, USA). Antibodies targeting PPARy2, C/EBPa, aP2 and $\beta$-actin were purchased from Santa Cruz Biotechnology (Santa Cruz, CA, USA). Protein expression was monitored by measurement of chemiluminescence alterations using Image Station 2000 MM (Eastman Kodak, Rochester, NY, USA). Quantitative analysis of these images was performed using Molecular Imaging Software Version 4.0 provided by Kodak 2000MM System.

\section{Statistical analysis}

The data are reported as the mean \pm standard deviation (SD). ANOVA (SPSS 13.0) was used for statistical analysis. Samples were considered normally distributed if $P>0.05$. Heterogeneity of variance was accepted if $P>0.05$, and the LSD method was used to perform appropriate pairwise comparisons. Otherwise, Tukey's post hoc test was adopted. The values were considered statistically significant at $P<0.05$.

\section{Results}

Effect of tanshinol on body weight during the experimental period

The body weight of all rats showed an ascending trend with age before the third week, especially in the Con group. Then, the body weight of rats in the GC group slowly decreased compared with the Con group until the experimental end point, but the body weight of rats in the Con group was maintained at the highest level and exhibited a relatively steady trend. Interestingly, the body weights in both the Tan group and Res group were higher than those in the GC group after the fourth week, especially after the eleventh week (Figure 2).

\section{Effect of tanshinol on GC-induced decreased bone mass and bone quality}

To verify the preventive influence of tanshinol on bone loss, we first scanned the trabecular bone using a $\mu-\mathrm{CT}$ machine and reconstructed a 3D-image of the bone micro-architecture. The data showed that GC contributed to the obviously damaged bone architecture in rats exposed to GC (Figure 3A and Table 


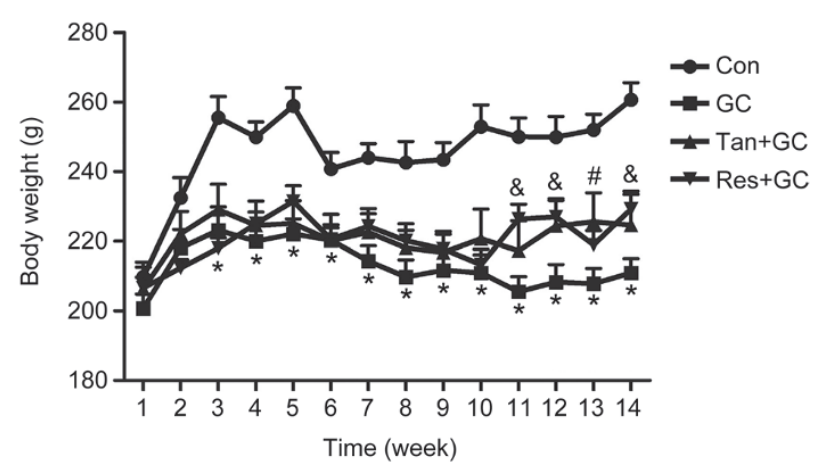

Figure 2. Alterations of the body weight of rats. The body weight of all rats in every group was recorded each week for 14 weeks. The data are presented as the mean $\pm \operatorname{SD}(n=8) .{ }^{*} P<0.05$ vs control group (Con). ${ }^{\#} P<0.05$, Tan plus GC $($ Tan $+G C)$ vs the model group $(\mathrm{GC}) ;{ }^{\circledR} P<0.05$, Res plus $\mathrm{GC}($ Res+GC) vs the model group $(G C)$.

2), which was in line with the significant histomorphometric parameters, including the decrease in percent trabecular bone area (\% Tb.Ar) and trabecular number (Tb.N) and increase in trabecular spacing (Tb.Sp). However, tanshinol exerted a sig- nificant protective effect on the bone mass of GIO rats (Figure $3 \mathrm{~B}, 3 \mathrm{C}$ and 3D). To further confirm whether treatment with tanshinol improved the biomechanical properties of bone, femoral shaft samples were assessed using the three-point bending test. The results showed that the fracture load and bending energy of rats in the GC group exhibited a significant reduction compared with those of rats in the Con group (Table 3). Expectedly, tanshinol attenuated the deleterious effects of GC on the biomechanical characteristics. Collectively, tanshinol contributed to the prevention of bone loss and improvement of bone strength in GIO rats, similar to the preventive effect of resveratrol.

\section{Effect of tanshinol on GC-triggered impaired bone formation}

We next asked whether the protective effect of tanshinol on bone was involved in restoring the impaired bone formation in rats exposed to GC. The representative histological images of calcein double-labeled trabeculae indicated that GC exerted a deleterious effect on cancellous bone in the distal femur, showing a smaller space between the calcein labels and weaker fluorescence intensity (Figure 4A). In accordance with the dual-fluorescence images, GC treatment led to a decrease

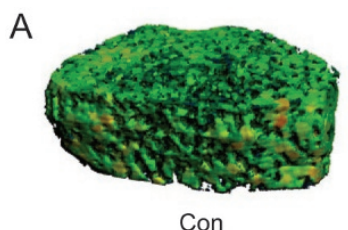

Con

B

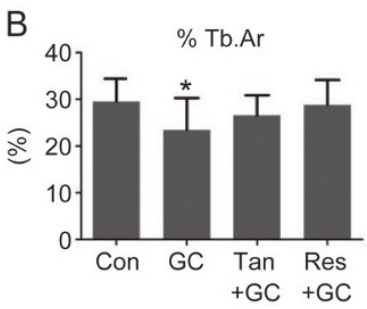

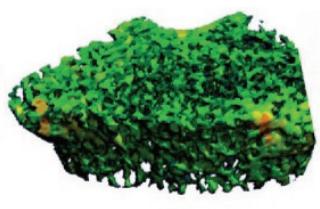

GC

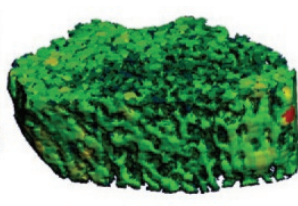

Tan+GC

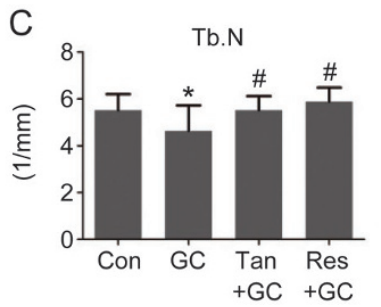

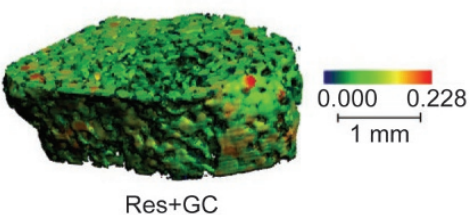

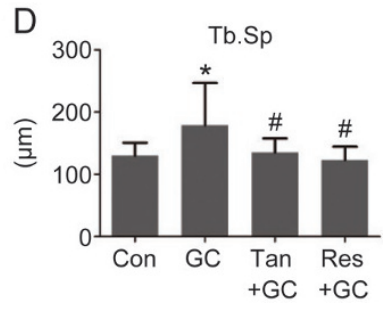

Figure 3. Tanshinol protects the bone micro-architecture against glucocorticoid. (A) The micro-architecture of the trabecular portion of the proximal femur of rats was measured and analyzed with a Micro-CT machine. The statistical parameters of cancellous bone in the proximal tibia of GIO rats were determined using histomorphometry: (B) trabecular area, (C) trabecular number and (D) trabecular spacing. The data are presented as the mean \pm SD $(n=8) .{ }^{*} P<0.05$ vs normal control (Con). ${ }^{\#} P<0.05$ vs GC treatment (GC).

Table 2. Effects of tanshinol on parameters for bone microstructures in GIO rats (mean $\pm \mathrm{SD}, n=8) .{ }^{*} P<0.05$ vs normal control (Con). ${ }^{*} P<0.05$ vs GC treatment (GC).

\begin{tabular}{lcccc}
\hline Parameters & Con & Group & Tan+GC & Res+GC \\
& $0.084 \pm 0.010$ & $0.070 \pm 0.012^{*}$ & $0.076 \pm 0.006$ & $0.081 \pm 0.011^{\#}$ \\
Tb.Th $(\mu \mathrm{m})$ & $6.81 \pm 1.21$ & $6.02 \pm 0.62$ & $7.92 \pm 0.67^{\#}$ & $7.73 \pm 0.87^{\#}$ \\
Tb.N $(1 / \mathrm{mm})$ & $0.44 \pm 0.06$ & $0.33 \pm 0.05^{*}$ & $0.41 \pm 0.04^{\#}$ & $0.45 \pm 0.07^{\#}$ \\
BV/TV $(\%)$ & $0.11 \pm 0.02$ & $0.13 \pm 0.02^{*}$ & $0.11 \pm 0.01^{\#}$ & $0.10 \pm 0.02^{\#}$ \\
Tb.Sp $(\mu \mathrm{m})$ & $0.05 \pm 0.80$ & $1.06 \pm 0.41^{*}$ & $0.25 \pm 0.34^{\#}$ & $-0.33 \pm 1.04^{\#}$ \\
SMl & $1.35 \pm 0.04$ & $1.41 \pm 0.06^{*}$ & $1.35 \pm 0.04^{\#}$ & $1.35 \pm 0.04^{\#}$ \\
DA & & & & \\
\hline
\end{tabular}


Table 3. Effects of tanshinol on biomechanical parameters of bone in GIO rats (mean \pm SD, $n=8$ ). ${ }^{*} P<0.05$ vs normal control (Con). ${ }^{\#} P<0.05$ vs GC treatment (GC).

\begin{tabular}{lcccc}
\hline Parameters & Con & Group & Tan+GC & Res+GC \\
& $143.70 \pm 14.80$ & $109.30 \pm 10.84^{*}$ & $124.59 \pm 19.24^{\#}$ & $132.88 \pm 11.25^{\#}$ \\
Elastic load (N) & $176.38 \pm 17.00$ & $161.37 \pm 21.91$ & $169.66 \pm 12.87$ & $176.26 \pm 10.16$ \\
$\begin{array}{l}\text { Fracture load (N) } \\
\text { Bending energy } \\
(\mathrm{N} \times \mathrm{mm})\end{array}$ & $14.63 \pm 2.63$ & $8.96 \pm 2.22^{*}$ & $12.39 \pm 3.22^{\#}$ & \\
Stiffness & & & $3.96 \pm 0.40$ & $3.92 \pm 0.54$ \\
$\left(\times 10^{4}, \mathrm{~N} \times \mathrm{mm}^{2}\right)$ & $4.06 \pm 0.53$ & $3.95 \pm 0.49$ & $3.65^{\#}$ \\
\hline
\end{tabular}

in the rate of bone formation (Figure $4 \mathrm{~B}$ and $4 \mathrm{C}$ ) and activity of mature osteoblasts (Figure 4D). Encouragingly, tanshinol exhibited a protective effect on the capacity of bone formation under GC treatment that was as effective as that of resveratrol (Figure 4). In short, these data demonstrated that tanshinol can protect bone formation from impairment induced by longterm excessive GC treatment.

Effect of tanshinol on GC-initiated changes in adipocyte distribution in the bone marrow

It is well-known that both bone formation and bone marrow adipogenesis are regulated by shared signaling pathways in bone marrow mesenchymal stem cells (MSCs). We next examined the adipocyte distribution in the bone marrow of rats treated with GC in the presence or absence of tanshinol or resveratrol. The results showed that GC caused an increase in the number and area of adipocytes in cancellous bone and marrow. Interestingly, tanshinol treatment led to fewer and a decreased area of adipocytes in the bone marrow than GC exposure without tanshinol treatment. Surprisingly, resveratrol had no significant influence on the number of adipocytes, but led to an increase in adipocyte area (Figure 5).
Effect of tanshinol on GC-elicited down-regulation of the KLF15/ PPARy2 pathway

It has been previously demonstrated that induction of adipocyte differentiation is dependent on subsequent activation of the KLF15/PPARy2 pathway ${ }^{[13]}$. To reveal if inhibition of the KLF15/PPARY2 pathway after treatment with tanshinol or resveratrol contributed to a decrease in adipogenesis, we monitored the expression levels of the KLF15 protein and PPARY2 protein in cancellous bone and marrow in response to GC, as well as mRNA expression of the KLF15, PPAR 2 2, C/EBPa and $a P 2$ genes. The data indicated that tanshinol treatment reversed up-regulation of KLF15 (Figure 6A and 6B), PPARY2 (Figure 6C and 6D), C/EBPa (Figure 6E and 6G) and aP2 (Figure 6F and 6G), which are necessary for blocking the GCinduced increase in adipogenesis (Figure 6). These findings demonstrate that tanshinol can block activation of the KLF15/ PPARY2 pathway elicited by GC, contributing to inhibition of the increased adipogenesis in cancellous bone and marrow. Notwithstanding the fact that the inhibitory effect of resveratrol on KLF15 expression was higher than that of tanshinol, resveratrol had little effect on the expression of $\mathrm{C} / \mathrm{EBPa}$ and $\mathrm{aP} 2$, in accordance with the findings regarding the number
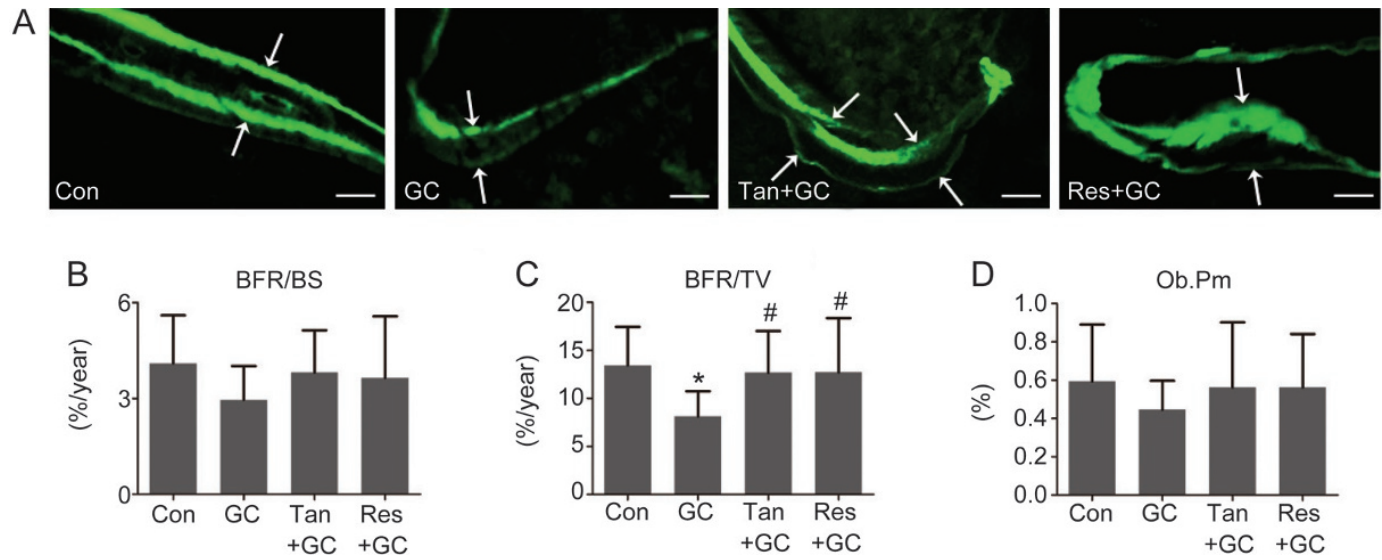

Figure 4. Tanshinol hampers the decreased bone formation induced by glucocorticoid. (A) Representative fluorescence micrographs of the tibia of rats showing the bone formation capacity detected with a dual calcein labeling assay. Bars: $100 \mu \mathrm{m}$. (B and C) The dynamic parameters of the rate of bone formation were determined by histomorphometry. (D) The statistical parameter of the osteoblast-lined bone surface was related to the activity of bone formation. The data are presented as the mean $\pm \mathrm{SD}(n=8)$. ${ }^{*} P<0.05$ vs normal control (Con). ${ }^{\#} P<0.05$ vs GC treatment (GC). 

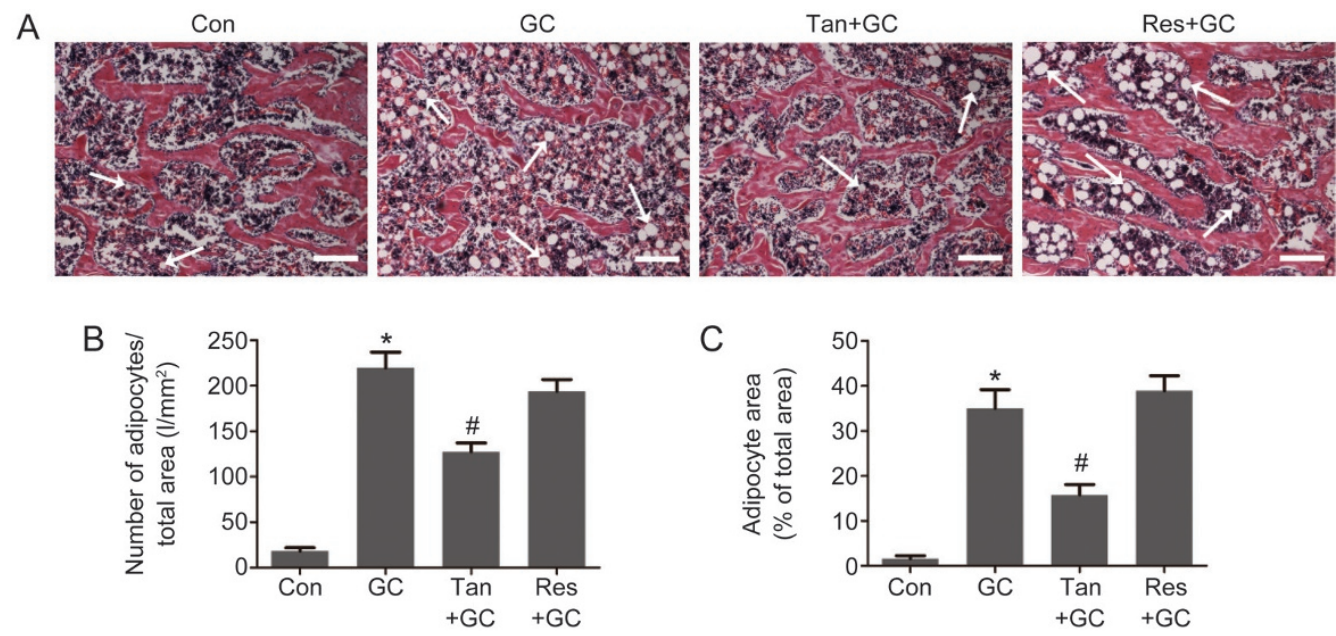

Figure 5. Tanshinol counteracts the increased marrow adiposity induced by glucocorticoid. (A) Representative images of H\&E staining demonstrating trabecular bone and marrow adipose tissue in the proximal tibia of rats. Bars: $100 \mu \mathrm{m}$. (B) Number of adipocytes over the total area and (C) adipocyte area over the total area were calculated using ImageJ 1.46r software. The data are presented as the mean \pm SD $(n=8)$. " $P<0.05$ vs normal control (Con). ${ }^{\#} P<0.05$ vs GC treatment (GC).

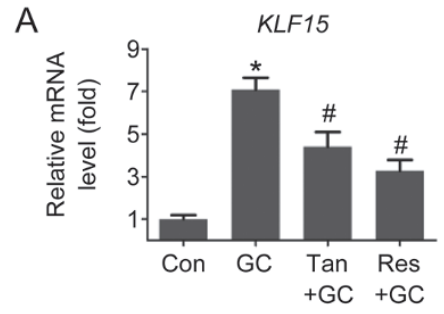

C

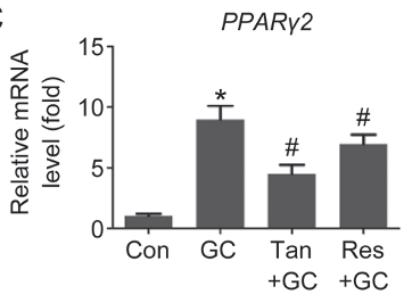

E

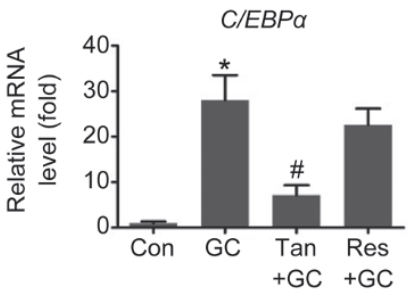

$\mathrm{F}$

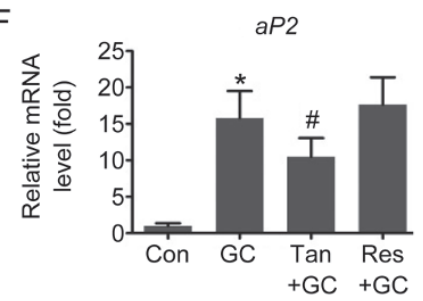

B

D
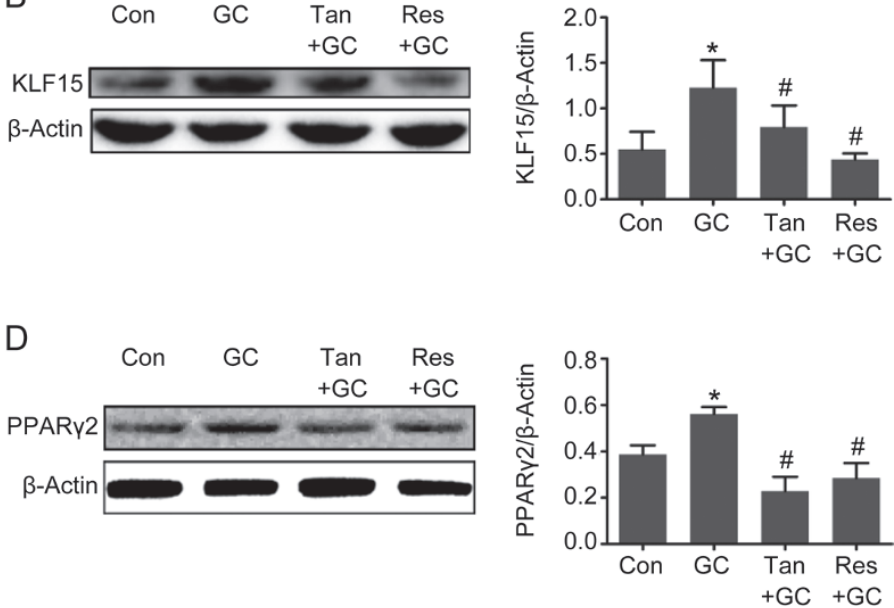

G
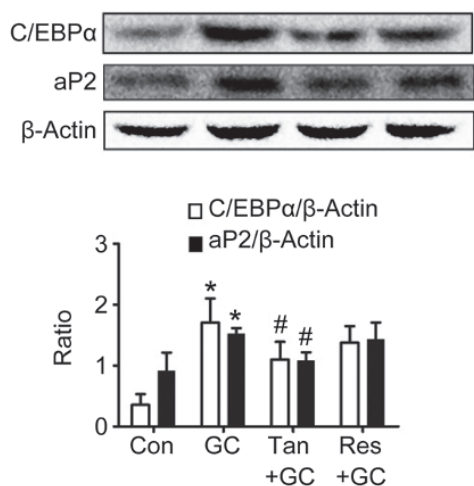

Figure 6. Tanshinol attenuates the up-regulation of the KLF15/PPARy2 pathway triggered by glucocorticoid. Expression of (A) KLF15 mRNA, (C) PPARY2 mRNA, (E) C/EBP $\alpha$ mRNA and (F) aP2 mRNA were measured with qRT-PCR. Expression of the (B) KLF15, (D) PPARY2, C/EBP $\alpha$ and aP2 (G) protein was determined by Western blot assay. The data are presented as the mean $\pm \mathrm{SD}(n=3) .{ }^{*} P<0.05$ vs normal control (Con). ${ }^{*} P<0.05$ vs GC treatment (GC). 
and area of adipocytes (Figure 6). These data suggest that tanshinol exerts preventive influence on increased adipocytes in GIO model rats via down-regulation of the KLF15/PPARY2 pathway.

\section{Effect of tanshinol on GC-induced alterations in Wnt/Fox03a signaling}

Based on the evidence listed above, we next asked whether tanshinol counteracted GC-elicited down-regulation of Wnt signaling that is required for bone formation. The evidence demonstrated that tanshinol hampered reduction of $\beta$-catenin protein (a key molecule in canonical Wnt signal transduction) and Axin2 mRNA (an indicator of the Wnt pathway) expression triggered by GC (Figure 7A and 7B). Simultaneously, we found that tanshinol counteracted the induction of the FoxO3a protein and Gadd45a mRNA (a FoxO3a-dependent gene), just like resveratrol (Figure 7C and 7D). In brief, the protective role of tanshinol on bone formation in rats exposed to GC involved up-regulation of Wnt signaling and down-regulation of the FoxO3a pathway.

\section{Discussion}

Well-documented evidence confirms that impaired bone formation is a significant characteristic of GIO. However, development of medication for GIO treatment is progressing slowly. Therefore, we explored tanshinol as a potential candidate for development as an anti-osteoporotic agent. In the present study, we demonstrated that tanshinol exerts a preventive effect on GIO through improvement of osteogenesis and inhibition of adipogenesis and further found that the mechanism underlying the inhibition of bone loss involves regulation of the KLF15/PPARY/FoxO3a/Wnt cascade pathways.

The findings of the present study confirmed that tanshinol can attenuate GC-triggered damage to the bone micro-architecture and poor bone quality, contributing to the prevention of osteoporosis (Figure 3 and Table 2). Furthermore, tanshinol counteracted GC-elicited impaired bone formation through stimulation of osteoblast differentiation, osteoblastic activity and ossification (Figure 4). In addition, tanshinol hampered GC-initiated marrow adipogenesis (Figure 5). These findings are, in part, in accordance with our previous in vitro and in vivo data ${ }^{[6,16,18,20,21]}$. Surprisingly, we observed that resveratrol caused an increased adipocyte area despite having no effect on the adipocyte number (Figure 5), which may be related to the promotion of adipocyte differentiation ${ }^{[22,23]}$. Despite the similar anti-osteoporotic effect of tanshinol and resveratrol, the causes of the differences in their effects on adipogenesis still need to be elucidated.

It is reasonable that cells exposed to long-term GC are prone to aging ${ }^{[24]}$. Actually, the level of GC is high in aged skeletons, and GC can mediate some adverse age-related changes in bone tissue $e^{[25,26]}$. In the present study, the mean body weight of rats in the GC group slowly decreased after the third week until the experimental end point compared with the relatively steady level observed in the Con group, but neither tanshinol or resveratrol reversed the decreasing trend in body weight triggered by GC (Figure 2). There are two possible explanations for these observations. From a metabolic viewpoint, GC directly leads to an increase in whole-body proteolysis and inhibition of glycogen synthesis ${ }^{[27,28]}$; in the process of skeletal growth, GC contributes indirectly to weight loss through inhibition of the growth of longitudinal bone and reduction of the whole-body bone mineral content ${ }^{[29]}$. As a result, GC as an inducer of aging may result in a decreased body weight due to apparent metabolic dysfunction, while tanshinol exerts a preventive effect on the reduction in body weight with aging.

Notwithstanding that fact that tanshinol blocked GCinduced marrow adiposity, we next investigated the signaling pathways related to adipogenesis. The findings demonstrated that tanshinol counteracts activation of KLF15 and PPARY2 elicited by GC in a model of GIO in rats and correspondingly leads to down-regulation of C/EBPa and aP2 (Figure 6). The
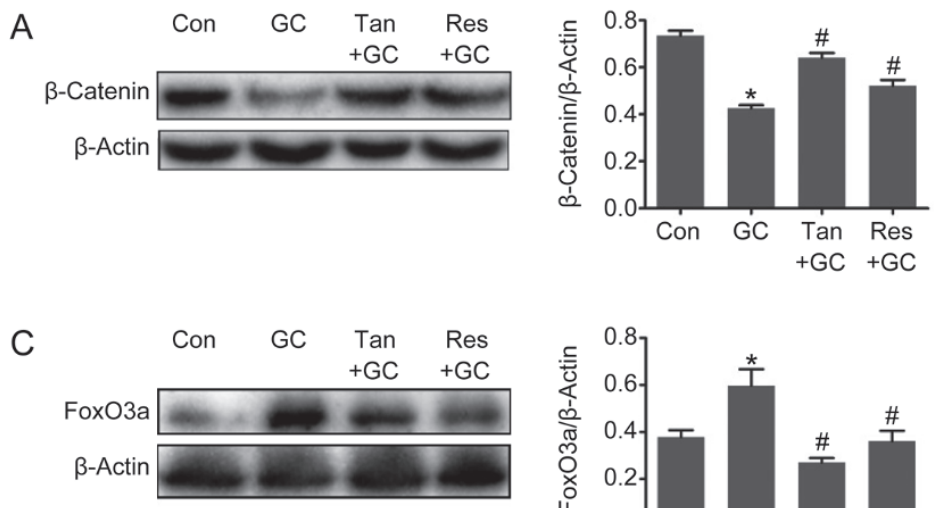
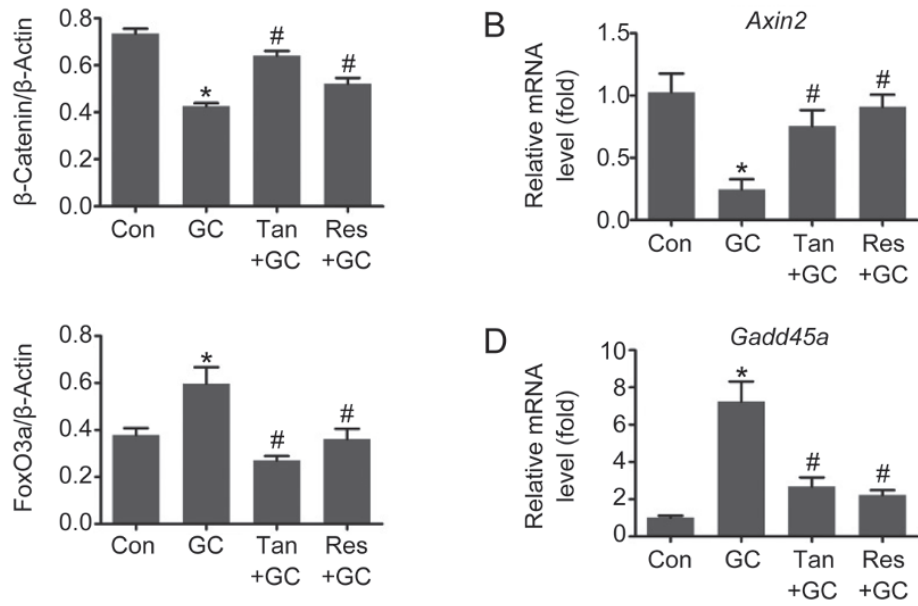

Figure 7. Tanshinol inhibits FoxO3a pathway activation and simultaneously reverses the down-regulation of Wnt signaling initiated by glucocorticoid. Expression of (A) FoxO3a protein and (C) $\beta$-catenin protein were determined by Western blot assay. Expression of (B) Axin2 and (D) Gadd45a mRNA was measured with qRT-PCR. The data are presented as the mean \pm SD $(n=3) .{ }^{*} P<0.05$ vs normal control (Con). ${ }^{\#} P<0.05$ vs GC treatment (GC). 
data also confirmed that resveratrol exerts an inhibitory effect on activation of KLF15 and PPARY2, but has no effect on expression of C/EBPa and aP2 (Figure 6), and the alterations of these two genes are similar to those of marrow adiposity (Figure 6). Generally, PPAPY in coordination with C/EBPa induces adipocyte-specific gene expression, including aP2, an adipocyte-specific protein that is used as a marker of adipogenesis differentiation ${ }^{[7,30]}$. Thus, the mechanism of resveratrol on adipogenesis in GIO model rats is not yet fully clear.

It is well-known that endogenous GC signaling in MSCs and osteoblasts is needed for bone remodeling. However, GC at supraphysiological (ie, pharmacological) concentrations has negative effects on bone formation. There is an increasing number of published literature reporting that osteoblasts and adipocytes share common MSCs in bone and that local and systemic pathologic factors in skeletal tissue may cause both bone loss and increased marrow adiposity in the development of osteoporosis ${ }^{[31]}$. Actually, a high dose of exogenous GC exerts an inhibitory effect on the $\mathrm{Wnt} / \beta$-catenin pathway in mature osteoblasts ${ }^{[26]}$ and simultaneously causes activation of PPARY signaling, which pushes MSCs toward differentiating into adipocytes and away from osteogenesis ${ }^{[32]}$. Notably, a previous study showed that KLF15 expression is increased in osteoblasts exposed to Dex ${ }^{[33]}$. Our previous findings further indicated that KLF15 activation in response to GC can suppress Wnt signaling in bone tissue ${ }^{[16]}$ and that KLF15 activation results in up-regulation of PPARY and FoxO3 $\mathrm{a}^{[11,17]}$. Our other previous study confirmed that activation of the FoxO3a transcription factor was at the expense of $\beta$-catenin/Tcf-mediated transcription, which contributes to inhibition of Wnt signaling $^{[18]}$. Importantly, our data confirmed that tanshinol attenuated inhibition of $\beta$-catenin protein and the axin2 (an indicator of the Wnt pathway) gene and simultaneously blocked expression of the FoxO3a protein and Gadd45 gene (a target gene of FoxO3a) under GC treatment conditions (Figure 7). Unfortunately, we did not verify the previous findings related to the fate of MSC differentiation when the KLF15 gene was knocked-in or knocked-out in vitro. Despite the preliminary aspect of the molecular mechanism assessment, this study clearly indicates that tanshinol exerts an anti-osteoporotic effect through adjustment of the imbalance between adipogenesis and osteogenesis triggered by long-term excessive GC treatment.

In summary, the present evidence indicates that tanshinol attenuates the GC-elicited increase in marrow adiposity and decrease in bone formation. Moreover, our findings suggest that the possible mechanism of tanshinol underlying its antiosteoporotic effect involves inhibition of the KLF15/PPARY2/ FoxO3a pathway and simultaneous stimulation of Wnt signaling. In addition, tanshinol is a water-soluble compound and has been commonly used for thousands of years as the main component of diverse complex prescriptions in Traditional Chinese Medicine. As a consequence, tanshinol might be able to be developed as an effective and safe candidate for prevention and treatment of GIO.

\section{Acknowledgements}

The study was supported by the National Natural Science Foundation of China (№ 81673814, 81273518), by the Science and Technology Planning Project of Guangdong Province (№ 2016A020215148), and by the Medical Scientific Research Foundation of Guangdong Province (№ A2016293).

\section{Author contribution}

Ya-jun YANG, Dong-tao WANG and Liao CUI participated in research design; Ya-jun YANG, Zhu ZHU, Dong-tao WANG, Xin-le ZHANG, Wen-xiu LAI, Yu-lin MO, Jin LI, Yan-long LIANG and Yong-jie YU performed experiments; Ya-jun YANG, Zhuo-qing HU and Yu-yu LIU analyzed the data; Yajun YANG, Dong-tao WANG and Wen-xiu LAI wrote or contributed to the writing of the manuscript.

\section{References}

1 Suzuki Y. Secondary osteoporosis. A review of recent evidence focusing on glucocorticoid-induced osteoporosis and osteoporosis associated with rheumatoid arthritis. Clin Calcium 2007; 17: 104-7.

2 Rizzoli R, Adachi JD, Cooper C, Dere W, Devogelaer JP, Diez-Perez A, et al. Management of glucocorticoid-induced osteoporosis. Calcif Tissue Int 2012; 91: 225-43.

3 Yao W, Cheng Z, Busse C, Pham A, Nakamura MC, Lane NE. Glucocorticoid excess in mice results in early activation of osteoclastogenesis and adipogenesis and prolonged suppression of osteogenesis: a longitudinal study of gene expression in bone tissue from glucocorticoid-treated mice. Arthritis Rheum 2008; 58: $1674-$ 86.

4 Gimble JM, Nuttall ME. The relationship between adipose tissue and bone metabolism. Clin Biochem 2012; 45: 874-79.

5 Mikami Y, Lee M, Irie S, Honda MJ. Dexamethasone modulates osteogenesis and adipogenesis with regulation of osterix expression in rat calvaria-derived cells. J Cell Physiol 2011; 226: 739-48.

6 Cui L, Li T, Liu Y, Zhou L, Li P, Xu B, et al. Salvianolic acid B prevents bone loss in prednisone-treated rats through stimulation of osteogenesis and bone marrow angiogenesis. PLoS One 2012; 7: e34647.

7 Cristancho AG, Lazar MA. Forming functional fat: a growing understanding of adipocyte differentiation. Nat Rev Mol Cell Biol 2011; 12: 722-34.

8 Zhuang H, Zhang X, Zhu C, Tang X, Yu F, Shang GW, et al. Molecular mechanisms of PPAR-gamma governing MSC osteogenic and adipogenic differentiation. Curr Stem Cell Res Ther 2016; 11: 25564.

9 Kawai M, Rosen CJ. PPARgamma: a circadian transcription factor in adipogenesis and osteogenesis. Nat Rev Endocrinol 2010; 6: 62936.

10 McConnell BB, Yang VW. Mammalian Kruppel-like factors in health and diseases. Physiol Rev 2010; 90: 1337-81.

11 Asada M, Rauch A, Shimizu H, Maruyama H, Miyaki S, Shibamori M, et al. DNA binding-dependent glucocorticoid receptor activity promotes adipogenesis via Kruppel-like factor 15 gene expression. Lab Invest 2011; 91: 203-15.

12 Ito S, Suzuki N, Kato S, Takahashi T, Takagi M. Glucocorticoids induce the differentiation of a mesenchymal progenitor cell line, ROB-C26 into adipocytes and osteoblasts, but fail to induce terminal osteoblast differentiation. Bone 2007; 40: 84-92.

13 Ahmed M, Gaffen SL. IL-17 inhibits adipogenesis in part via C/ 
EBPalpha, PPARgamma and Kruppel-like factors. Cytokine 2013; 61: 898-905.

14 Hossain MG, Iwata T, Mizusawa N, Shima SWN, Okutsu T, Ishimoto $\mathrm{K}$, et al. Compressive force inhibits adipogenesis through COX-2mediated down-regulation of PPARy2 and C/EBP $\alpha$. J Biosci Bioeng 2010; 109: 297-303.

15 Graneli C, Karlsson C, Brisby H, Lindahl A, Thomsen P. The effects of PPAR-gamma inhibition on gene expression and the progression of induced osteogenic differentiation of human mesenchymal stem cells. Connect Tissue Res 2014; 55: 262-74.

16 Yang Y, Su Y, Wang D, Chen Y, Liu Y, Luo S, et al. Tanshinol rescues the impaired bone formation elicited by glucocorticoid involving in KLF15 pathway. Oxid Med Cell Longev 2016: 1092746. doi: 10.115/2016/1092746.

17 Shimizu N, Yoshikawa N, Ito N, Maruyama T, Suzuki Y, Takeda S, et al. Crosstalk between glucocorticoid receptor and nutritional sensor mTOR in skeletal muscle. Cell Metab 2011; 13: 170-82.

18 Yang Y, Su Y, Wang D, Chen Y, Wu T, Li G, et al. Tanshinol attenuates the deleterious effects of oxidative stress on osteoblastic differentiation via Wnt/FoxO3a signaling. Oxid Med Cell Longev 2013; 2013.

19 Lam FF, Yeung JH, Chan KM, Or PM. Relaxant effects of danshen aqueous extract and its constituent danshensu on rat coronary artery are mediated by inhibition of calcium channels. Vascul Pharmacol 2007; 46: 271-7.

20 Cui L, Liu YY, Wu T, Ai CM, Chen HQ. Osteogenic effects of D+beta3,4-dihydroxyphenyl lactic acid (salvianic acid A, SAA) on osteoblasts and bone marrow stromal cells of intact and prednisone-treated rats. Acta Pharmacol Sin 2009; 30: 321-32.

21 Luo S, Yang Y, Chen J, Zhong Z, Huang H, Zhang J, et al. Tanshinol stimulates bone formation and attenuates dexamethasone-induced inhibition of osteogenesis in larval zebrafish. J Orthop Transl 2016; 4: 35-45.

$22 \mathrm{Hu}$ P, Zhao L, Chen J. Physiologically achievable doses of resveratrol enhance 3T3-L1 adipocyte differentiation. Eur J Nutr 2015; 54: 56979.

23 Wang S, Liang X, Yang Q, Fu X, Rogers CJ, Zhu M, et al. Resveratrol induces brown-like adipocyte formation in white fat through activation of AMP-activated protein kinase (AMPK) [alpha]1. Int J Obes 2015; 39: 967-76.

24 Zannas AS, Arloth J, Carrillo-Roa T, lurato S, Roh S, Ressler KJ, et al. Lifetime stress accelerates epigenetic aging in an urban, African American cohort: relevance of glucocorticoid signaling. Genome Biol 2015; 16: 266.

25 Almeida M, Han L, Ambrogini E, Weinstein RS, Manolagas SC. Glucocorticoids and tumor necrosis factor alpha increase oxidative stress and suppress Wnt protein signaling in osteoblasts. J Biol Chem 2011; 286: 44326-35.

26 Seibel MJ, Cooper MS, Zhou H. Glucocorticoid-induced osteoporosis: mechanisms, management, and future perspectives. Lancet Diabetes Endocrinol 2013; 1: 59-70.

27 Simon D, Alberti C, Alison M, Le Henaff L, Chevenne D, Boizeau P, et al. Effects of recombinant human growth hormone for 1 year on body composition and muscle strength in children on long-term steroid therapy: randomized controlled, delayed-start study. J Clin Endocrinol Metab 2013; 98: 2746-54

28 Liu XY, Shi JH, Du WH, Fan YP, Hu XL, Zhang CC, et al. Glucocorticoids decrease body weight and food intake and inhibit appetite regulatory peptide expression in the hypothalamus of rats. Exp Ther Med 2011; 2: 977-84.

29 Lin S, Huang J, Zheng L, Liu Y, Liu G, Li N, et al. Glucocorticoidinduced osteoporosis in growing rats. Calcif Tissue Int 2014; 95 : 362-73.

30 Shan T, Liu W, Kuang S. Fatty acid binding protein 4 expression marks a population of adipocyte progenitors in white and brown adipose tissues. FASEB J 2013; 27: 277-87.

31 Devlin MJ, Rosen CJ. The bone-fat interface: basic and clinical implications of marrow adiposity. Lancet Diabetes Endocrinol 2015; 3: 141-7.

32 Colaianni G, Brunetti G, Faienza MF, Colucci S, Grano M. Osteoporosis and obesity: role of Wnt pathway in human and murine models. World J Orthop 2014; 5: 242.

33 Rauch A, Seitz S, Baschant U, Schilling AF, Illing A, Stride B, et al. Glucocorticoids suppress bone formation by attenuating osteoblast differentiation via the monomeric glucocorticoid receptor. Cell Metab 2010; 11: 517-31. 